\title{
Tissue Kidney Injury Molecule-1 Expression in the Prediction of Renal Function for Several Years after Kidney Biopsy
}

\author{
Sanja Simic Ogrizovic, ${ }^{1,2}$ Suzana Bojic, ${ }^{3}$ Gordana Basta-Jovanovic, ${ }^{4}$ Sanja Radojevic, ${ }^{4}$ \\ Jelena Pavlovic, ${ }^{1}$ Jelena Kotur Stevuljevic, ${ }^{5}$ Violeta Dopsaj, ${ }^{5}$ and Radomir Naumovic ${ }^{1,2}$ \\ ${ }^{1}$ Clinic of Nephrology, Clinical Center of Serbia, Pasterova 2, 11000 Belgrade, Serbia \\ ${ }^{2}$ School of Medicine, University of Belgrade, Serbia \\ ${ }^{3}$ Clinical Hospital Center Bezanijska Kosa, Belgrade, Serbia \\ ${ }^{4}$ Institute of Pathology, School of Medicine, University of Belgrade, Belgrade, Serbia \\ ${ }^{5}$ Faculty of Pharmacy, University of Belgrade, Belgrade, Serbia
}

Correspondence should be addressed to Sanja Simic Ogrizovic; ssogrizovic@gmail.com

Received 30 June 2013; Accepted 7 October 2013

Academic Editor: Giuseppe Murdaca

Copyright (C) 2013 Sanja Simic Ogrizovic et al. This is an open access article distributed under the Creative Commons Attribution License, which permits unrestricted use, distribution, and reproduction in any medium, provided the original work is properly cited.

Objectives. Retrospective study was designed to examine the importance of tissue kidney injury molecule-1 (KIM-1) expression in predicting kidney function in sixty patients $(27$ males) aged $34.15 \pm 12.23$ years with different kidney diseases over three years after kidney biopsy. Materials and Methods. Tissue KIM-1 expression was determined immunohistochemically and KIM-1 staining was scored semiquantitatively, as well as tubulointerstitialis (TIN), inflammation, atrophy, and fibrosis. Kidney function (MDRD formula) and proteinuria/day were evaluated at the time of biopsy (GFR0) and 6, 12, 24, and 36 months later. Results. Significantly positive correlations between tissue KIM-1 expression and age $(r=0.313)$, TIN inflammation $(r=0.456)$, fibrosis $(r=0.317)$, and proteinuria at 6 months $(r=0.394)$ as well as negative correlations with GFR0 $(r=-0.572)$, GFR6 $(r=-0.442)$, GFR24 $(r=-0.398)$, and GFR36 ( $r=-0.412)$ were found. Meanwhile, TIN inflammation was the best predictor of all measured kidney functions during three years, while tissue KIM-1 expression $(P=0.016)$ was a predictor only at 6 months after biopsy. Conclusion. Tissue KIM-1 expression significantly predicts kidney function solely at 6 months after biopsy, when the effects of immune and nonimmune treatments are the strongest.

\section{Introduction}

Chronic kidney disease (CKD) is associated with a number of comorbidities and prognosis is poor, because many patients experience progression to end-stage renal disease [1]. Also, in the majority of patients disease progression may be altered due to more or less suitable immunosuppressive protocol treatments and therapeutic approaches. The mechanisms of injury underlying progression are blurred, but traditional opinion pointed to an association between decline in renal function with the degree of proteinuria and histological findings of glomerulosclerosis and interstitial fibrosis $[2,3]$.

Kidney injury molecule-1 (KIM-1) is a new specific biomarker of proximal tubule injury that can be measured both in urine and kidney tissue samples. It is an apoptotic-cell phagocytosis and scavenger receptor that is most highly upregulated in proximal tubular epithelium in acute and chronic kidney injury [4]. Also, much attention has been paid to its possible pathophysiological role in modulation of tubular damage and repair [5-7].

Besides a close relationship between tissue KIM-1 expression and urinary KIM-1 concentration, our previous sixmonth prospective pilot study showed that KIM-1 expression in tissue correlated better with TIN features and renal function in different chronic kidney diseases than with urinary KIM-1 concentration [8]. Therefore, that investigation was the basis of this long-term retrospective study, where only KIM-1 tissue expression as a predictor of long-term kidney function was examined.

The aim of this retrospective investigation was to evaluate (1) possible associations between tissue KIM-1 expression and tubulointerstitial (TIN) inflammation, atrophy, 
and fibrosis in different chronic kidney diseases and (2) possible associations between tissue KIM-1 expression and some demographic and laboratory parameters as well as kidney function and proteinuria at the time of biopsy and 6 , 12,24 , and 36 months later.

\section{Methods}

2.1. Patients. The retrospective study included 60 patients $(27$ men) of mean age $34.42 \pm 12.15$ years (range $18-59$ years), who were hospitalized in the Clinical Center of Serbia for kidney biopsy from 2006 to 2009. Indications for kidney biopsy were nephrotic syndrome, pathologic proteinuria without nephrotic syndrome, and abnormal urinary sediment (erythrocyturia and leukocyturia) in several samples.

Pathohistological analysis revealed minimal change glomerulonephritis (MCGN) in two patients, non-IgA GN in nine, IgA GN in six, membranous and membranoproliferative GN in seven patients each, focal glomerulosclerosis in eleven, lupus nephritis in ten, and crescentic GN in eight patients. After diagnosis, the participants were treated according to established protocols for each type of GN with immunological and nonimmunological therapy (nonsalt diets, statins, angiotensin-converting-enzyme-ACE inhibitors, and angiotensin receptor type 1 blockers ATB).

The investigation was planned according to the ethical guidelines of the Declaration of Helsinki. The institutional review committee approved our study protocol therebys following local biomedical research regulations (IRB No. 877/7).

2.2. Laboratory Methods. A complete blood count; serum urea and creatinine, albumin; lipid status, and urine sediment were determined in blood and urine samples collected 1 day before kidney biopsy as well proteinuria/day. Only samples with a sterile urine culture were processed. Urine sediments with more than $3 \mathrm{RBC} / \mathrm{hpf}$ or $5 \mathrm{WBC} / \mathrm{hpf}$ were defined as clinically significant erythrocyturia or leukocyturia. Estimated glomerular filtration rate (eGFR) was calculated with a shortened version of the Modification of Diet in Renal Disease (MDRD; patients $\geq 18$ years) [9].

2.2.1. Morphological Changes. Alteration in TIN were assessed after routine hematoxylin-eosin staining by a qualified nephropathologist who was not familiar with the preset histopathologic or clinical diagnosis, nor the identity of patients. Inflammation activity was then scored numerically (0: no inflammation, 1: slightly marked inflammation, 2: moderate inflammation, and 3: strong inflammation) as well as atrophy and fibrosis advancement (0: no atrophy and no fibrosis, 1: slightly marked atrophy and fibrosis, 2: moderate atrophy and fibrosis, 3: very pronounced atrophy and fibrosis).

2.2.2. Kim-1 Expression in Tissue. After deparaffinization tissue samples were rehydrated and stained immunohistochemically for KIM-1 using the tissue KIM-1/TIM-1 kit ( $\mathrm{R}$ \& D Systems Inc., Minneapolis, MN, USA) with contrasting
TABLE 1: Tissue samples scoring system based on percentage of KIM1 positive tubules.

\begin{tabular}{lc}
\hline \% KIM-1 positive tubules & Score \\
\hline 0 & 0 \\
$0.1-12.5$ & 0.5 \\
$12.6-25.0$ & 1 \\
$25.1-50.0$ & 2 \\
$50.1-75.0$ & 3 \\
$75.1-100$ & 4 \\
\hline
\end{tabular}

hematoxylin and eosin staining. A tubule was considered to be KIM-1 positive if it contained at least one KIM-1 positive cell regardless of whether the KIM-1 expression was cytoplasmic or apical. KIM-1 staining was scored semiquantitatively by estimating the percentage of cortical tubules expressing KIM-1 per field (the complete biopsy area was scored, with a minimum of five fields; in controls 30 fields were scored). Then the score was assigned to each tissue sample as shown in Table 1 [10]. Unaffected parts of kidneys from patients with renal cell carcinoma were used as the negative control.

The 6-, 12-, 24-, and 36-month followup for all patients included measurement of serum creatinine concentration, eGFR, and proteinuria.

2.3. Statistics. Data are presented as mean \pm 1 standard deviation (SD) and the range of values. The one-sample Kolmogorov-Smirnov test was used to check for normal distribution of the variables. Relationships between variables were estimated using Pearson's rank and Spearmen's correlation tests as appropriate. Correlations or differences were considered statistically significant if two-tailed $P<$ 0.05. Independent predictors of renal function $6,12,24$, and 36 months after the kidney biopsy were identified by stepwise multivariant regression analysis. All calculations were performed using SPSS software.

\section{Results}

Table 2 presents the demographic, clinical, and laboratory data for the patients at the time of kidney biopsy.

Tissue KIM-1 expression was significantly induced in all kidney biopsies except in one patient with MCGN. Tissue KIM-1 had a typically apical localization, but it could also be found in the cytoplasm with no apparent affinity for the apical membrane of tubulocytes. KIM-1 positive tubules were located mainly in the TIN regions affected by inflammation or fibrosis but were not observed in regions with end-stage fibrosis. KIM-1 positive tubules were often dilated cortical tubules, sometimes with initial signs of atrophy. Completely atrophic tubules were KIM-1 negative (Figure 1).

Univariate analysis showed significant positive associations between tissue KIM-1 expression and age $(r=0.313$; $P=0.015)$, TIN inflammation $(r=0.4563 ; P=0.004)$, TIN fibrosis $(r=0.317 ; P=0.021)$, and hemoglobin $(r=-0.440$; $P=0.001)$ as well as negative associations with albumin concentration $(r=-0.376 ; P=0.011)$ and eGFR $(r=-0.572$; 


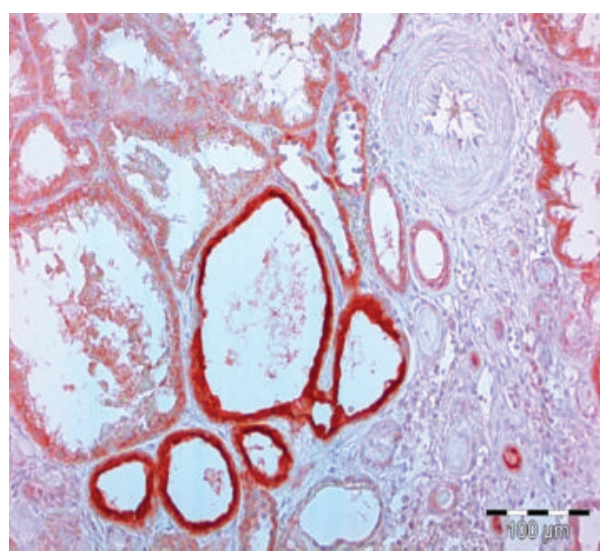

(a)

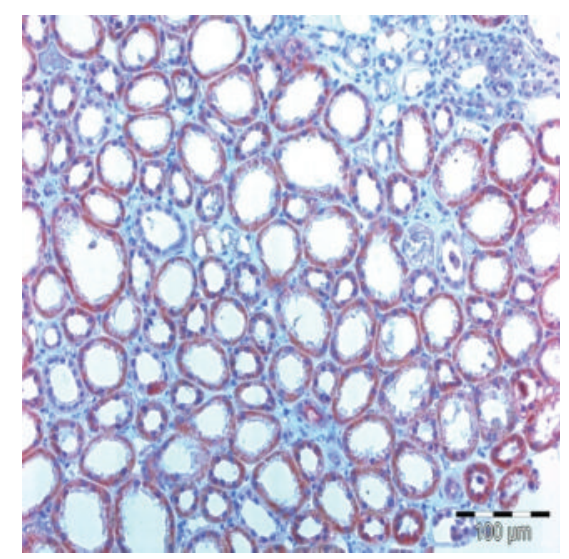

(b)

FIGURE 1: KIM-1 positive tubules are of intense red color. (a) Apical membrane of tubulocytes has the strongest affinity for KIM-1 specific antibodies. (b) Entire cytoplasm of tubulocytes has strong affinity for KIM-1 specific antibodies.

TABLE 2: Demographic, clinical, and laboratory data of patients at the time of kidney biopsy.

\begin{tabular}{lc}
\hline Variables & $X \pm \mathrm{SD}$ \\
\hline Age (years) & $34.42 \pm 12.15$ \\
Gender $(\mathrm{M} / \mathrm{F})$ & $27 / 33$ \\
MAP $(\mathrm{mmHg})$ & $98.55 \pm 10.17$ \\
Hemoglobin $(\mathrm{g} / \mathrm{L})$ & $122.40 \pm 27.28$ \\
Albumin $(\mathrm{g} / \mathrm{L})$ & $29.08 \pm 9.73$ \\
Serum creatinine $(\mu \mathrm{mol} / \mathrm{L})$ & $185.50 \pm 186.28$ \\
eGFR $\left(\mathrm{mL} / \mathrm{min} / 1.73 \mathrm{~m}^{2}\right)$ & $58.39 \pm 35.93$ \\
Proteinuria $(\mathrm{g} /$ day $)$ & $5.25 \pm 4.36$ \\
\hline
\end{tabular}

MAP: mean arterial pressure; eGFR: estimated glomerular filtration rate.

$P<0.001)$ at the time of biopsy and $6(r=-0.442 ; P=$ $0.002), 24(r=-0.398 ; P=0.012)$, and $36(r=-0.412$; $P=0.015)$ months later. Tissue KIM-1 expression correlated significantly only with proteinuria/day 6 months after biopsy $(r=0.394 ; P=0.026)$ but not with proteinuria at the time of biopsy and 12, 24, and 36 months later.

Table 3 gives the correlation coefficients between eGFR at the time of biopsy and 6,12, 24, and 36 months later and the examined variables. Multivariant stepwise regression analysis showed that the best predictor of kidney function at the time of biopsy (eGFR 0$)$ was TIN inflammation $(P<0.001)$, as well as of eGFR12 $(P=0.015)$, eGFR $24(P=0.039)$, and eGFR $36(P=0.012)$. However tissue KIM-1 expression was the best predictor of eGFR $6(P=0.016)$ along with TIN inflammation $(P=0.016)$ (Table 4$)$. Figure 2 presents the linear correlation between TIN inflammation $(P<0.001)$, tissue KIM-1 expression $(P<0.001)$, and eGFR 6.

\section{Discussion}

In our previous 6-month prospective study, we found higher urine KIM-1 concentrations in twenty chronic renal patients than in control subjects and a significant positive correlation between urine KIM-1 level and KIM-1 expression in tissue [8]. Although, urine KIM-1 content reflected tissue KIM-1 expression, tissue KIM-1 correlated better with TIN inflammation and fibrosis as well as with kidney function at the time of biopsy and 3 and 6 months later. Therefore, we retrospectively examined sixty patients with different kidney diseases in order to evaluate the importance of tissue KIM-1 expression in predicting kidney function in the ensuing 36 months after kidney biopsy.

In the present study tissue KIM-1 expression was associated with both TIN inflammation activity and TIN fibrosis. In addition, KIM-1 positive tubules were located in parts of the cortex that were imbued with inflammatory infiltrate and/or fibrotic changes but not in completely atrophic areas. It is well known that the expression of KIM-1 in tubules is associated with inflammation and TIN damage [11]. Doublelabeling studies have shown simultaneous expression of KIM1 and markers of prefibrotic changes, repair, and chemotaxis $[10,11]$. The study of van Timmeren et al. [10] also demonstrated a connection between KIM-1 expression in tissue, interstitial fibrosis, and macrophage accumulation. Ichimura et al. [7] first showed that tubular epithelial cells expressing KIM-1 on their surface could act as phagocytes for apoptotic and necrotic renal epithelial cells. KIM-1 is expressed predominantly in renal tubules, which, judging by the simultaneous expression of markers of dedifferentiation [10], are currently under the influence of agents that cause injury, but is absent from completely atrophic tubules. Controversy remains about the function of KIM-1: is it actively regulating the inflammatory process? or is its expression just a response to damage, attempted recovery, and/or repair? [11].

The present study revealed a significant negative correlation between tissue KIM-1 expression and all estimated GFR at the time of kidney biopsy and 6, 24, and 36 months later. However multivariate analysis pointed to TIN inflammation, but neither TIN fibrosis nor proteinuria, as the best predictor of kidney function at biopsy and 6, 12, 24, and 36 months afterwards. However, along with TIN inflammation, 
TABLE 3: Correlation coefficients between eGFR at the time of biopsy and 6, 12, 24, and 36 months later and measured variables.

\begin{tabular}{|c|c|c|c|c|c|}
\hline & eGFR 0 & eGFR 6 & eGFR 12 & eGFR 24 & eGFR 36 \\
\hline Age & -0.273 & - & - & - & - \\
\hline KIM-1 tissue expression & -0.572 & -0.340 & - & -0.378 & -0.374 \\
\hline TIN atrophy & -0.444 & -0.305 & -0.403 & - & - \\
\hline TIN inflammation & -0.713 & -0.309 & -0.434 & -0.369 & -0.518 \\
\hline TIN fibrosis & -0.378 & -0.381 & -0.352 & - & - \\
\hline Prt 0 & - & - & - & - & - \\
\hline Prt 6 & & - & - & -0.575 & -0.511 \\
\hline
\end{tabular}

TIN: tubulo-interstitialis; eGFR 0: estimated glomerular filtration rate at the time of biopsy; eGFR 6: estimated glomerular filtration rate 6 months after biopsy; eGFR 12: estimated glomerular filtration rate 12 months after biopsy; eGFR 24: estimated glomerular filtration rate 12 months after biopsy; eGFR 24: estimated glomerular filtration rate 24 months after biopsy; eGFR 36: estimated glomerular filtration rate 36 months after biopsy.

Prt 0: proteinuria/day at the time of biopsy; prt 6: proteinura/day 6 months after biopsy; prt 12: proteinuria/day 12 months after biopsy.

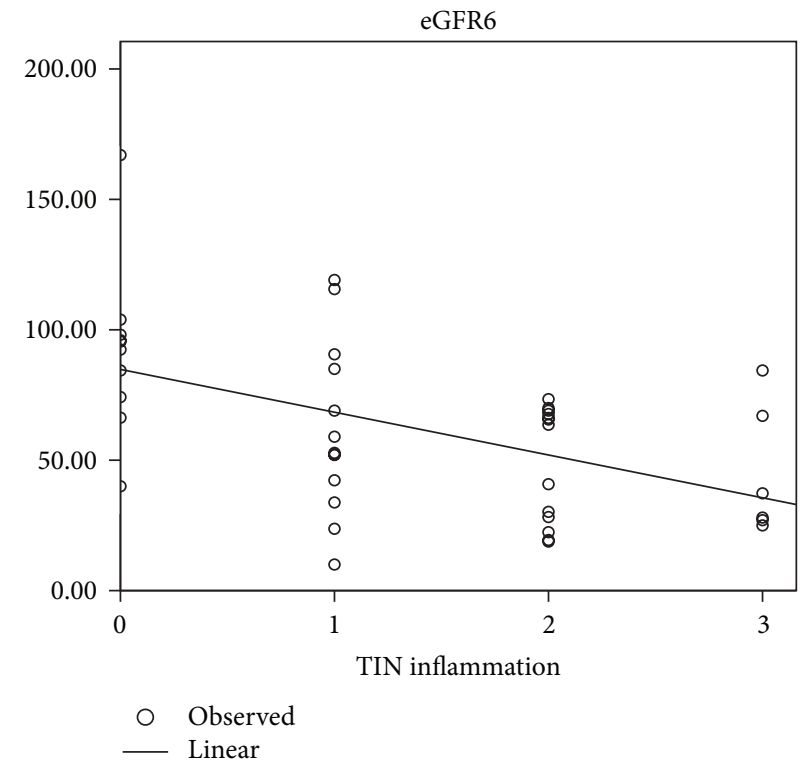

(a)

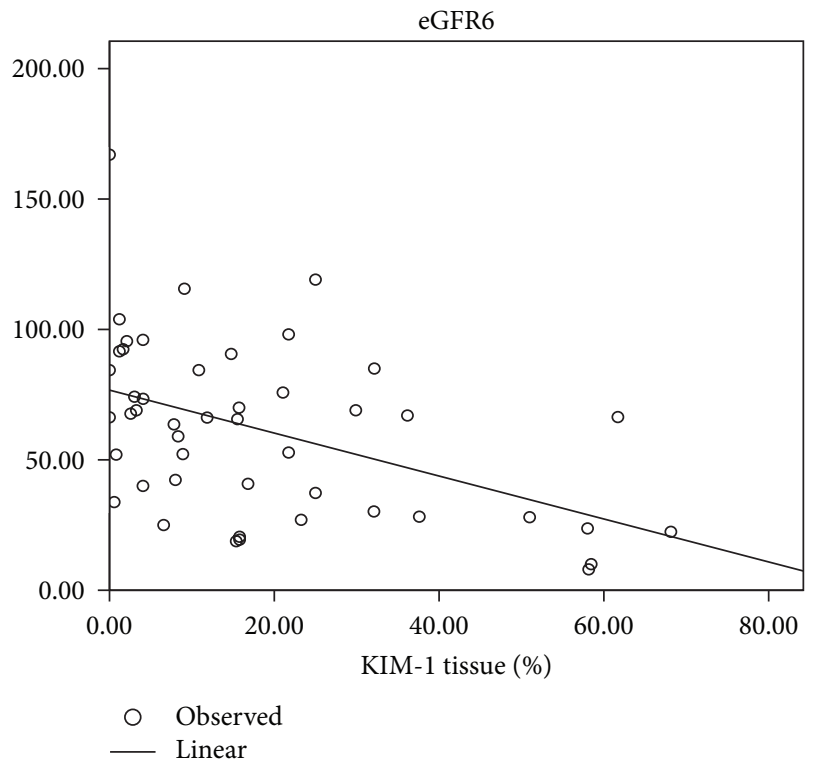

(b)

FIGURE 2: Linear correlation between TIN inflammation $(P=0.000)$, tissue KIM-1 expression $(P=0.000)$, and eGFR 6 months after kidney biopsy.

tissue KIM-1 expression was the best predictor of kidney function 6 months after biopsy.

Interstitial infiltration of inflammatory cells can be seen in a variety of immune and nonimmune kidney diseases and is thought to play a significant role in TIN damage and fibrosis. Upon exposure to high protein concentrations, renal tubule cells produce a host of chemokines, vasoactive mediators, and adhesion molecules, which may contribute to interstitial fibrosis [12]. Protocol immunosuppressive therapy and treatment with ACE inhibitors and AT blockers for chronic renal patients with different chronic immune and nonimmune-mediated kidney disorders proven by biopsy have the strongest effects during the first 6 months of therapy. The severity of pretreatment TIN damage predicts a dulled response to renoprotective intervention, with a worse long-term renal outcome [13]. Therefore, we can speculate that patients with less interstitial inflammation at the time of kidney biopsy will have a more favorable response to treatment and better kidney function afterwards. Also, the finding that KIM-1 was the strongest predictor of kidney function 6 months after biopsy along with TIN inflammation may support the hypothesis about its potential role in the development of interstitial fibrosis and an unsatisfactory treatment response.

Association between higher urinary KIM-1 levels and poorer kidney function was found in some human studies in native $[10,14]$ and transplanted kidneys [15] but data on human tissue KIM-1 expression are scarce. Zhang et al. [16] found that KIM-1 expression in transplant biopsies is a sensitive measure of cell injury. In addition, they showed that more intense KIM-1 staining predicts a better graft outcome over the ensuing 18 months and speculated that its level of expression may be an indicator of graft function recovery.

It is well known that proteinuria is an ominous biomarker of progressive kidney disease [2] and our study revealed that only proteinuria 6 months after kidney biopsy correlated 
TABLE 4: Results of stepwise multivariate regression analysis with estimated glomerular filtration rate 6 months after biopsy as dependant variable.

\begin{tabular}{|c|c|c|c|c|c|}
\hline \multicolumn{6}{|c|}{ Coefficients $^{\mathrm{a}}$} \\
\hline \multirow{2}{*}{ Model } & \multicolumn{2}{|c|}{ Unstandardized coefficients } & \multirow{2}{*}{$\begin{array}{c}\text { Standardized coefficients } \\
\text { Beta }\end{array}$} & \multirow{2}{*}{$t$} & \multirow{2}{*}{ Sig. } \\
\hline & B & Std. error & & & \\
\hline TIN inflammation & -15.878 & 4.482 & -.493 & -3.542 & .001 \\
\hline TIN inflammation & -11.763 & 4.511 & -.366 & -2.607 & .013 \\
\hline KIM1 tissue percent & -.650 & .259 & -.353 & -2.515 & .016 \\
\hline
\end{tabular}

${ }^{\mathrm{a}}$ Dependent variable: eGFR 6.

TIN: tubulointerstitialis; eGFR6: estimated glomerular filtration rate 6 months after biopsy.

negatively with kidney function 24 and 36 months after the biopsies and positively with KIM-1 expression in tissue. Earlier investigations $[10,14,17]$ have yielded conflicting data regarding the relationship between urinary KIM-1 level or tissue KIM-1 expression and proteinuria. One explanation may be that proteinuria is not always accompanied by TIN damage and a progressive decline in renal function $[18$, 19]. Data regarding the selectivity of proteinuria were not available for the purposes of this study.

\section{Conclusion}

The present study exposed a strong association between KIM1 tissue staining and TIN inflammation and fibrosis but not with TIN atrophy. Although multivariate analysis pointed to TIN inflammation, as the best predictor of kidney function at biopsy and 6, 12, 24, and 36 months later, tissue KIM-1 expression is one of the best predictor of kidney function 6 months after biopsy, the time when treatment effects are the strongest. Therefore, we can speculate that KIM-1 has a potential role in the development of interstitial fibrosis and poor treatment responses.

The major limitation of this investigation is the relatively small number of patients examined but it could be the basis for a larger prospective follow-up study for evaluation of urinary KIM-1 concentration and tissue KIM-1 expression in patients with various chronic and acute renal diseases.

\section{Conflict of Interests}

All the authors have declared no competing interests.

\section{Acknowledgment}

This work was supported by a grant from the Ministry of Science and Technological Development, Republic of Serbia (Project no. 175089).

\section{References}

[1] F. Kronenberg, "Emerging risk factors and markers of chronic kidney disease progression," Nature Reviews Nephrology, vol. 5, no. 12, pp. 677-689, 2009.

[2] K. Zandi-Nejad, A. A. Eddy, R. J. Glassock, and B. M. Brenner, "Why is proteinuria an ominous biomarker of progressive kidney disease?” Kidney International. Supplement, vol. 66, no. 92, pp. S76-S89, 2004.

[3] B. Rodríguez-Iturbe, R. J. Johnson, and J. Herrera-Acosta, "Tubulointerstitial damage and progression of renal failure," Kidney International. Supplement, vol. 68, no. 99, pp. S82-S86, 2005.

[4] T. Ichimura, C. R. Brooks, and J. V. Bonventre, "Kim-1/Tim-1 and immune cells: shifting sands," Kidney International, vol. 81, no. 9, pp. 809-811, 2012.

[5] G. J. Ko, D. N. Grigoryev, D. Linfert et al., "Transcriptional analysis of kidneys during repair from AKI reveals possible roles for NGAL and KIM-1 as biomarkers of AKI-to-CKD transition," The American Journal of Physiology - Renal Physiology, vol. 298, no. 6, pp. F1472-F1483, 2010.

[6] T. Ichimura, J. V. Bonventre, V. Bailly et al., "Kidney injury molecule-1 (KIM-1), a putative epithelial cell adhesion molecule containing a novel immunoglobulin domain, is up-regulated in renal cells after injury," The Journal of Biological Chemistry, vol. 273, no. 7, pp. 4135-4142, 1998.

[7] T. Ichimura, E. J. Asseldonk, B. D. Humphreys, L. Gunaratnam, J. S. Duffield, and J. V. Bonventre, "Kidney injury molecule-1 is a phosphatidylserine receptor that confers a phagocytic phenotype on epithelial cells," The Journal of Clinical Investigation, vol. 118, no. 5, pp. 1657-1668, 2008.

[8] S. Simic Ogrizovic, S. Bojic, G. Basta Jovanovic, J. Kotur Stevuljevic, V. Dopsaj, and V. Lezaic, "Kidney injury molecule-1 (KIM1) determination in tissue and urine of patients with different kidney disease," in Proceedings of the 50th ERA-EDTA Congress Abstracts2View, 2011, http://www.abstracts2view.com/era.

[9] A. S. Levey, J. P. Bosch, J. B. Lewis, T. Greene, N. Rogers, and D. Roth, "A more accurate method to estimate glomerular filtration rate from serum creatinine: a new prediction equation. Modification of diet in renal disease study group," Annals of Internal Medicine, vol. 130, no. 6, pp. 461-470, 1999.

[10] M. M. van Timmeren, M. C. van den Heuvel, V. Bailly, S. J. Bakker, H. van Goor, and C. A. Stegeman, "Tubular kidney injury molecule-1 (KIM-1) in human renal disease," The Journal of Pathology, vol. 212, no. 2, pp. 209-217, 2007.

[11] F. Waanders, M. M. van Timmeren, C. A. Stegeman, S. J. Bakker, and H. van Goor, "Kidney injury molecule-1 in renal disease," The Journal of Pathology, vol. 220, no. 1, pp. 7-16, 2010.

[12] T. W. Meyer, "Tubular injury in glomerular disease," Kidney International, vol. 63, no. 2, pp. 774-787, 2003.

[13] A. B. Kramer, H. Bos, H. van Goor, and G. J. Navis, "Sodium intake modifies the negative prognostic value of renal damage prior to treatment with ACE inhibitors on proteinuria induced by adriamycin," Nephron, vol. 103, no. 1, pp. p43-p52, 2006.

[14] H. P. E. Peters, F. Waanders, E. Meijer et al., "High urinary excretion of kidney injury molecule-1 is an independent predictor 
of end-stage renal disease in patients with IgA nephropathy," Nephrology Dialysis Transplantation, vol. 26, no. 11, pp. 35813588, 2011.

[15] M. M. van Timmeren, V. S. Vaidya, R. M. van Ree et al., "High urinary excretion of kidney injury molecule-1 is an independent predictor of graft loss in renal transplant recipients," Transplantation, vol. 84, no. 12, pp. 1625-1630, 2007.

[16] P. L. Zhang, L. I. Rothblum, W. K. Han, T. M. Blasick, S. Potdar, and J. V. Bonventre, "Kidney injury molecule-1 expression in transplant biopsies is a sensitive measure of cell injury," Kidney International, vol. 73, no. 5, pp. 608-614, 2008.

[17] X. Zhao, Y. Zhang, L. Li et al., "Glomerular expression of kidney injury molecule-1 and podocytopenia in diabetic glomerulopathy," The American Journal of Nephrology, vol. 34, no. 3, pp. 268280, 2011.

[18] G. D’Amico and C. Bazzi, "Pathophysiology of proteinuria," Kidney International, vol. 63, no. 3, pp. 809-825, 2003.

[19] C. Bazzi, C. Petrini, V. Rizza, G. Arrigo, and G. D’Amico, “A modern approach to selectivity of proteinuria tubulointerstitial damage in nephrotic syndrome," Kidney International, vol. 58, no. 4, pp. 1732-1741, 2000. 


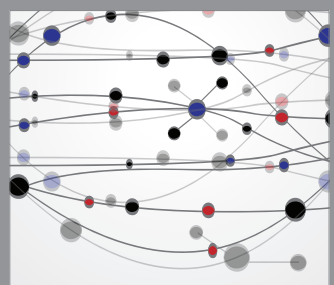

The Scientific World Journal
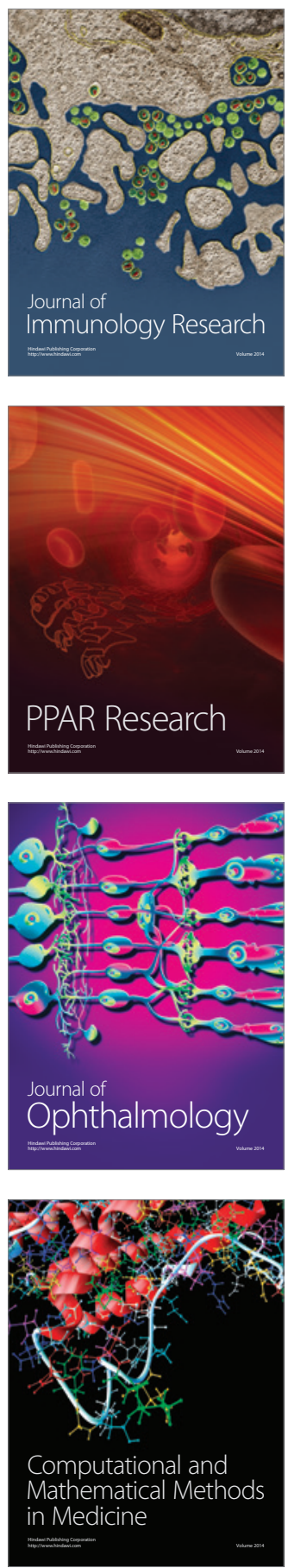

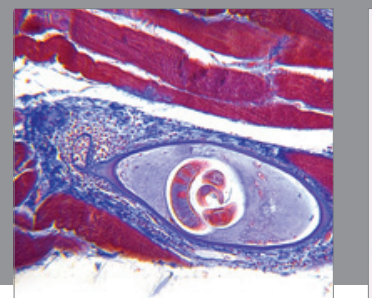

Gastroenterology

Research and Practice
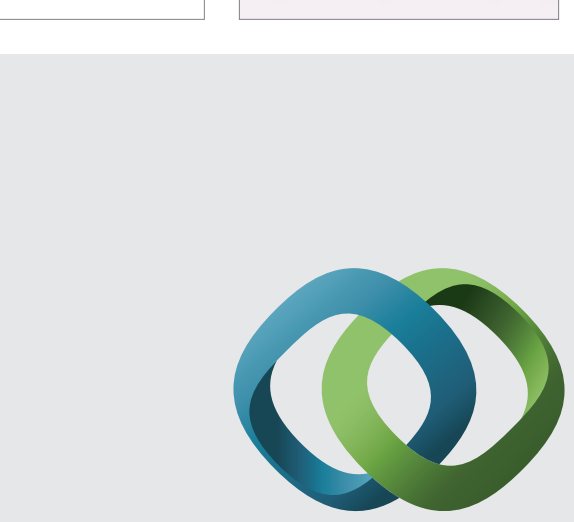

\section{Hindawi}

Submit your manuscripts at

http://www.hindawi.com
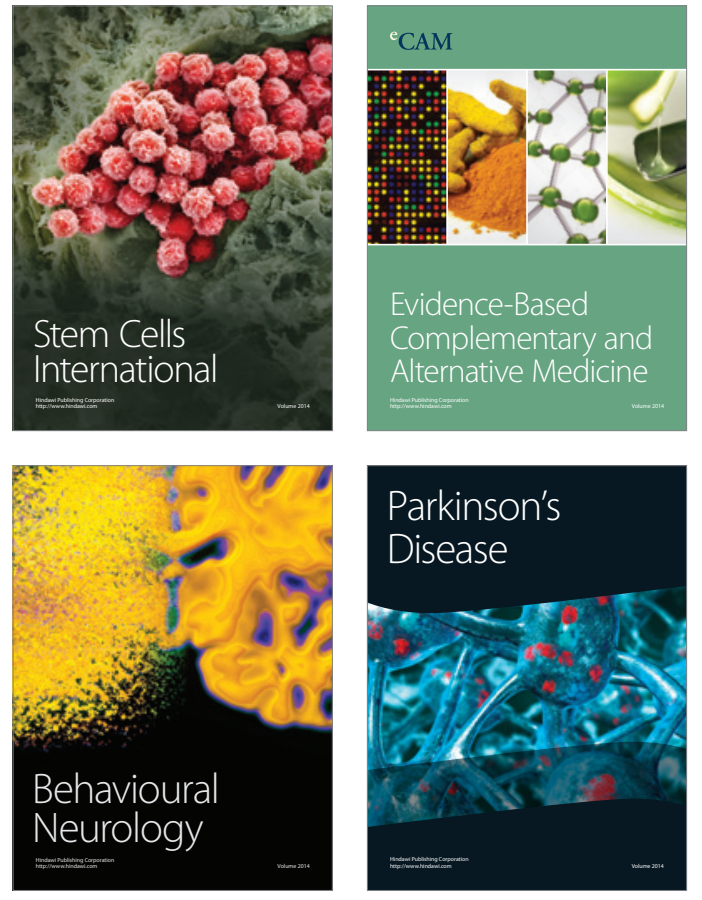
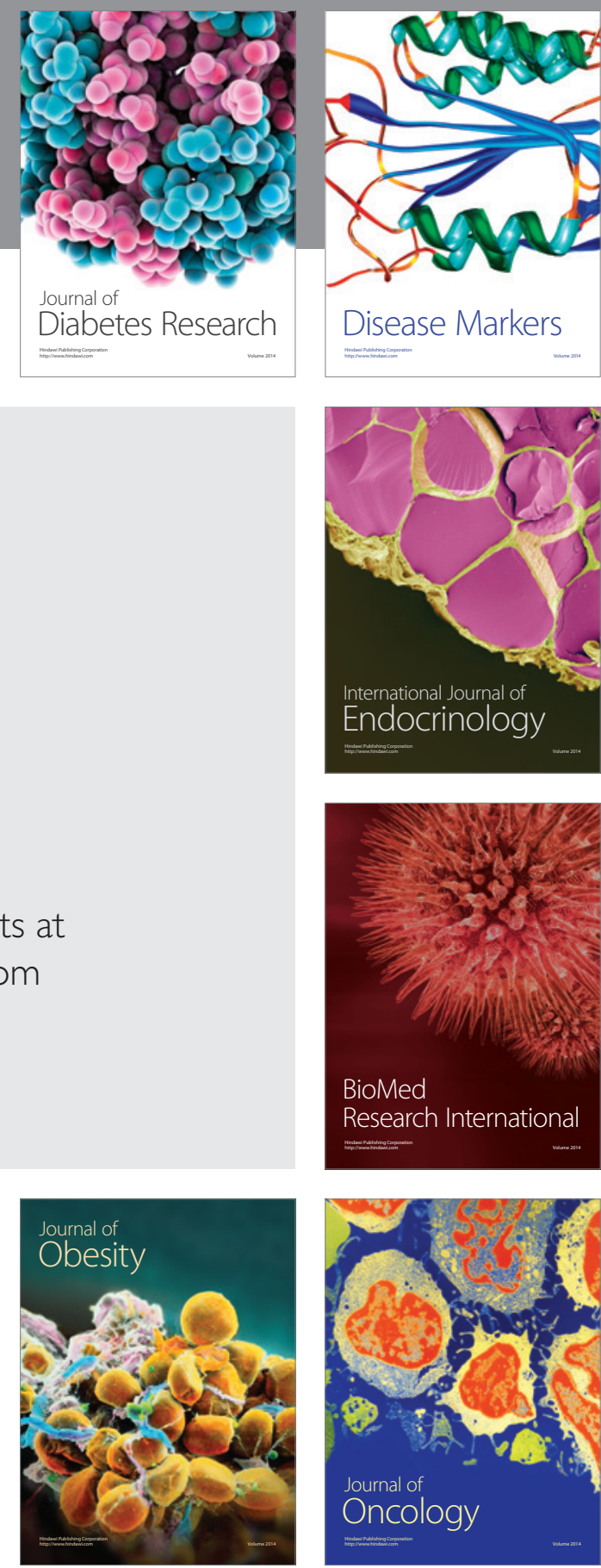

Disease Markers
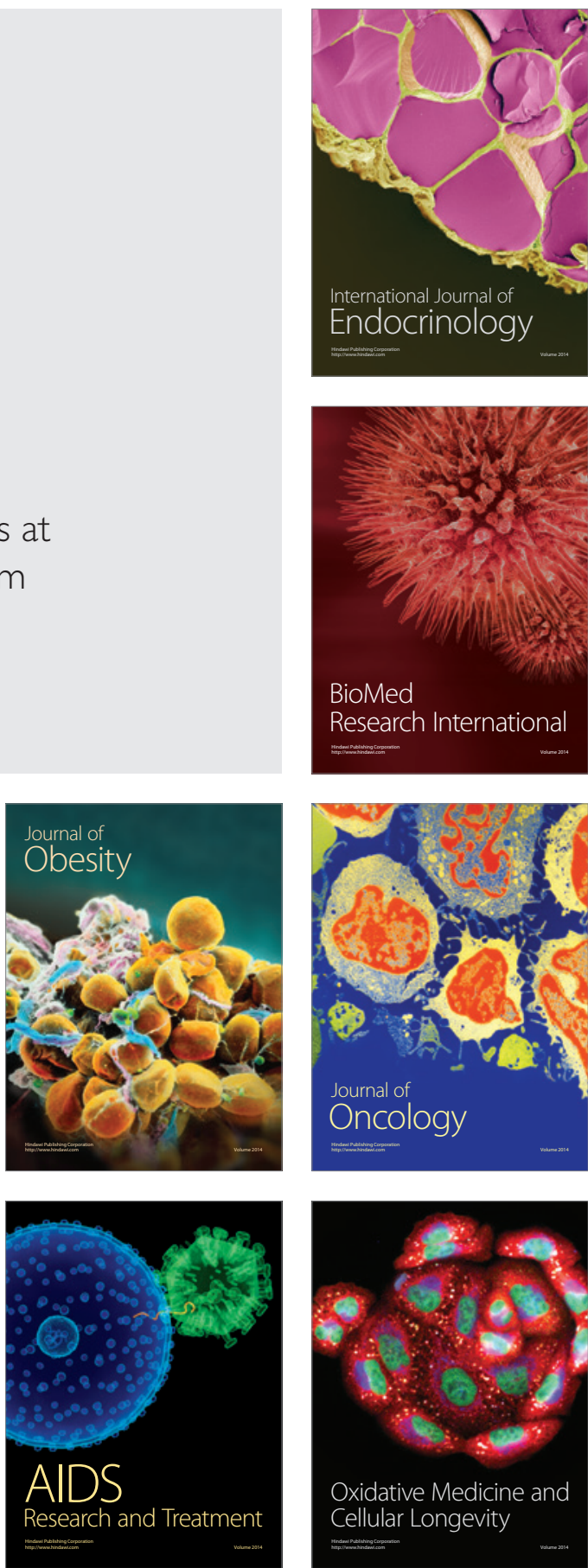